\title{
A novel method to isolate mesenchymal stem cells from mouse umbilical cord
}

\author{
BIN ZHANG $^{1 *}$, JIE ZHANG $^{2 *}$, HUI SHI $^{1}$, FEI MAO $^{1}$, JUANJUAN WANG $^{1}$, YONGMIN YAN $^{1}$, \\ XU ZHANG ${ }^{1}$, HUI QIAN ${ }^{1}$ and WENRONG XU ${ }^{1,3}$ \\ ${ }^{1}$ Key Laboratory of Medical Science and Laboratory Medicine, School of Medicine, Jiangsu University, Zhenjiang, \\ Jiangsu 212013; ${ }^{2}$ Department of Laboratory Medicine, Affiliated Hospital of Nanjing University of \\ Chinese Medicine, Jiangsu Hospital of Traditional Chinese Medicine, Nanjing, Jiangsu 210029; ${ }^{3}$ Department of \\ Laboratory Medicine, The Affiliated Hospital, Jiangsu University, Zhenjiang, Jiangsu 212000, P.R. China
}

Received August 22, 2016; Accepted May 11, 2017

DOI: $10.3892 / \mathrm{mmr} .2017 .7950$

\begin{abstract}
Mesenchymal stem cells (MSCs), derived from various tissues, are considered an ideal cell source for clinical use, among which MSCs from the umbilical cord exhibit advantages over those from adult tissues. In preclinical studies, mouse models and xenogeneic MSC treatment are most commonly used to imitate diseases and clinical practice, respectively. However, the efficiency of cross-species therapy remains controversial, making it difficult to elucidate the underlying mechanisms. Thus, allogeneic therapy may be more instructive and meaningful in clinical use. To confirm this hypothesis, the present study established a novel method for the isolation and expansion of MSCs from mouse umbilical cords (mUC-MSCs) to support in vivo experiments in mice. MSCs were isolated from mUCs and mouse bone marrow (mBM), and then identified by flow cytometry. The differences in mUC-MSCs and mBM-MSCs were analyzed using a growth curve and their differentiation ability. The results showed that the harvested cells exhibited general characteristics of MSCs and possessed the capacity for long-term culture. Despite having similar morphology and surface antigens to MSCs derived from mouse bone marrow, the mUC-MSCs showed differences in purification, proliferation, stem cell markers and differentiation. In addition to detailed characterization, the present study verified the presence of Toll-like receptor 3 (TLR3), an important component of immune responses, in
\end{abstract}

Correspondence to: Professor Wenrong Xu or Professor Hui Qian, Key Laboratory of Medical Science and Laboratory Medicine, School of Medicine, Jiangsu University, 301 Xuefu Road, Zhenjiang, Jiangsu 212013, P.R. China

E-mail: icls@ujs.edu.cn

E-mail:1stmmmlst@163.com

*Contributed equally

Key words: mesenchymal stem cells, umbilical cord, mouse, Toll-like receptor
mUC-MSCs. It was found that the activation of TLR3 upregulated the levels of stemness-related proteins, and enhanced the secretion and mRNA levels of inflammatory cytokines in the pre-treated mUC-MSCs. Collectively, the results of the present study provide further insight into the features of newly established mUC-MSCs, providing novel evidence for the selection of murine MSCs and their responses to TLR3 priming.

\section{Introduction}

In the late 20th century, there was significant progress in stem cell research when mesenchymal stem cells (MSCs) were first identified and named $(1,2)$. MSCs are fibroblast-like pluripotent adult cells, which possess the following characteristics making them ideal candidates for cell-based therapy: i) Readily expanded from adult and fetal tissues; ii) multilineage capabilities (plasticity); iii) immune privileged; iv) has immunomodulatory abilities; v) releases trophic factors; and vi) exhibits homing to damaged sites (3). MSCs have been successfully isolated from various tissues, including bone marrow (BM), umbilical cord (UC), adipose tissue, placenta, skin, muscle and tonsil tissues (3-6).

Adult tissue-derived MSCs, for example BM-MSCs, can have limitations in cell number and increase the risk of age-related changes, whereas younger tissue-derived MSCs, for example UC-MSCs, are increasing in popularity for clinical use due to their faster rate of self-renewal and higher differentiation potential. In addition, UC-MSCs share a similar gene expression profile to embryonic stem cells, but without the ethical issues and risk of tumorigenesis (7-10). The isolation of UC-MSCs has been performed in humans (3), horses (11), dogs (12), pigs (13), rats (14) and mice (9), of which mouse UC-derived MSCs (mUC-MSCs) have been reported only once.

An increasing number of studies have verified the therapeutic effects of UC-MSCs in clinical trials $(15,16)$ and preclinical studies, amongst which animal models are crucial in translating the in vitro properties of MSCs into therapeutic applications and examining mechanisms of efficacy. There is encouraging evidence from various experimental models indicating that UC-MSCs function across species 
barriers, including in renal ischemia-reperfusion injury (17), diabetes (18), Huntington's disease (12) and mammary carcinoma (14). However, studies have reported conflicting results in cross-species models, with xenogeneic MSCs showing detrimental effects (19). MSCs from different tissues may exhibit different intrinsic properties. Mice and humans are the most commonly used recipient and donnor species, however, certain key effector molecules are divergent between murine MSCs (mMSCs) and human MSCs (hMSCs), including nitric oxide and indoleamine 2,3-dioxygenase (20). Therefore, allogeneic MSC therapy may be more suitable for preclinical studies.

Toll-like receptors (TLRs) are considered to be an important family of conserved receptors, which mediate immune responses upon activation by pathogen components or endogenous molecules. Accumulating evidence indicates that MSCs from different species (mouse and human) express functional TLRs, and that their activation affects MSC functions, including proliferation, migration, differentiation and immunomodulation $(21,22)$. In addition, Waterman et al $(23,24)$ described MSC polarization into two phenotypes by TLR signaling. Specifically, TLR4-primed hMSCs (MSC1) exhibited a pro-inflammatory profile and attenuated tumor growth, whereas TLR3-primed MSCs (MSC2) expressed immunosuppressive mediators and promoted tumor growth. Few studies have investigated mMSCs and TLRs together, and whether TLR3 or TLR4 with specific ligands control mMSC functions remains to be elucidated.

To date, the most commonly used mMSCs in mouse models are mBM-MSCs. The establishment of mUC-MSCs, novel members of the mMSC bank and promising candidates for allogeneic cell therapy, may be an important platform for elucidating the cellular and molecular mechanisms of diseases (9). In the present study, mUC-MSCs derived from Kunming mice were successfully isolated and expanded using a novel method and culture system. The isolated cells were characterized in comparison with mBM-MSCs, and the effects of TLR3 on the expression of stemness-related proteins and cytokines in the mUC-MSCs were investigated. The results may provide novel clues for selecting ideal mMSCs in various mouse models and offer novel insights into the role of TLR3 as a regulator of mUC-MSCs.

\section{Materials and methods}

Mice. Breeding pairs of Kunming mice were purchased from the Laboratory Animal Research Center of Jiangsu University (Jiangsu, China). The total number of mice used in the present study was 12 and the ratio of males to females was 3:1 per cage (age, 8 weeks old; weight, 21 g; 9 males; 3 females). All animals were housed at $20-26^{\circ} \mathrm{C}$ with a relative humidity of $40-70 \%$; the feeding box was $1-2^{\circ} \mathrm{C}$ higher than the environment, with $5-10 \%$ humidity. A $12-\mathrm{h}$ light/dark cycle was also used and they had free access to food and water. Experimental procedures involving animals were approved by the Institutional Animal Care Committee of Jiangsu University and performed in strict accordance with the guidelines and regulations.

mUC-MSC isolation and expansion. Fresh mouse UCs were aseptically collected from Kunming mice at a gestational age of 15-19 days. The collected UCs were rinsed with phosphate-buffered saline (PBS) containing penicillin and streptomycin. The washed tissues were mechanically cut into small sections $\left(0.5-1.0 \mathrm{~mm}^{3}\right)$ and suspended in Dulbecco's modified Eagle's medium: F-12 nutrient mixture (F12/DMEM; Hyclone Laboratories; GE Healthcare Life Sciences, Logan, UT, USA) containing $15 \%$ fetal bovine serum (FBS; Gibco; Thermo Fisher Scientific, Inc., Waltham, MA, USA), $100 \mathrm{U}$ penicillin and streptomycin. The tissue sections, together with culture medium, were seeded into $3.5 \mathrm{~mm}$ cell culture dishes (Corning Incorporated, Corning, NY, USA) and incubated at $37^{\circ} \mathrm{C}$ in humidified air with $5 \% \mathrm{CO}_{2}$. The medium was replaced every 3 days, and the non-adherent tissues and cells were removed. When well-developed colonies of heterogeneous primary cells had formed at 9-12 days, the attached cells were trypsinized using $0.25 \%$ trypsin-EDTA (Invitrogen; Thermo Fisher Scientific, Inc.) and passaged. The first passage was performed at a ratio of 4:1-2:1, followed by successive passages at a ratio of 1:1-1:2 for early passages (passages 3-5), with medium being replaced every 2 days. The homogenously fibroblast-like cell populations appeared following five passages. mUC-MSCs at passage numbers 7-12 were used for the follow-up experiments.

Isolation and expansion of $m B M-M S C s$. The mBM-MSCs were isolated and cultured using protocols recommended previously (4). Briefly, 4-6 week old male mice (weight, $>30 \mathrm{~g}$ ) were sacrificed and fresh bone marrow cells were harvested by flushing the femurs with PBS, and cultured in low glucose DMEM (Gibco; Thermo Fisher Scientific, Inc.) containing $15 \% \mathrm{FBS}$ at $37^{\circ} \mathrm{C}$ in humid air with $5 \% \mathrm{CO}_{2}$. Following the initial 3 days, culture medium replacement was performed once every 3 days. On reaching confluence, the cells in colonies were trypsinized and subcultured. MSCs at passages 15-18 were used for biological characterization.

Flow cytometric analysis. For surface marker analysis, the MSCs were detached and stained with anti-mouse monoclonal antibodies for $30 \mathrm{~min}$ at $4^{\circ} \mathrm{C}$, including PE-conjugated $\mathrm{CD} 11 \mathrm{~b}$ (cat no. 12-0112-82; eBioscience, San Diego, CA, USA), CD44 (cat no. 553134), CD45 (cat no. 553081); FITC-conjugated CD29 (cat no. 561796), and CD34 (cat no. 560238; BD Pharmingen, San Diego, CA, USA). The labeled cells were analyzed using flow cytometry (FACSCalibur; BD Biosciences, San Jose, CA, USA). PE-IgG and FITC-IgG were used as controls; for all antibodies, $1 \mu \mathrm{l}$ antibody was added to $2.5 \times 10^{5}$ cell suspension in $200 \mu \mathrm{l}$ PBS. The analysis of the DNA content of the MSCs was performed as described above. The cell pellets were incubated in propidium iodide on ice for $30 \mathrm{~min}$. The percentages of cells in the G0/G1, G2/M and S phases were analyzed using flow cytometry using BD CellQuest ${ }^{\mathrm{TM}}$ Pro software (BD Biosciences).

Growth curve analysis. The MSCs were seeded in 24-well plates $\left(5.0 \times 10^{3}\right.$ cells/well) during the logarithmic growth phase. The number of cells per well was counted for 7 days using an inverted microscope (Nikon Corporation, Tokyo, Japan), and the procedure on each day was repeated three times.

Osteogenic and adipogenic differentiation in vitro. To induce osteogenic differentiation, the MSCs were seeded at $4 \times 10^{4}$ cells 
in $3.5 \mathrm{~cm}$ culture dishes, and cultured in osteogenic induction medium consisting of F12/DMEM or low glucose-DMEM with $15 \%$ FBS and osteogenic supplements $\left(10^{-8} \mathrm{M}\right.$ dexamethasone, $10 \mathrm{mM} \beta$-glycerophosphate and $50 \mathrm{mg} / 1$ ascorbic acid; Sigma-Aldrich; Merck Millipore, Darmstadt, Germany) at $37^{\circ} \mathrm{C}$, with the medium replaced every 3 days. The cells were induced for four cycles, and then subjected to alkaline phosphatase staining, which was observed using a light microscope (Nikon Corporation).

To evaluate adipogenic potential, MSCs at a density of $2 \times 10^{5}$ cells/dish were cultured in adipogenic induction medium A and B consisting of F12/DMEM or low glucose-DMEM with $15 \%$ FBS. Medium A was supplemented with $1 \mu \mathrm{M}$ dexamethasone, $200 \mu \mathrm{M}$ indomethacin and $2 \mu \mathrm{M}$ insulin (Sigma-Aldrich; Merck Millipore) whereas medium B was supplemented with $2 \mu \mathrm{M}$ insulin. Medium A was replaced every 3 days, followed by maintaining in medium B for 1 day. The cells were induced for two cycles, and then identified using Oil red $\mathrm{O}$ staining, which was observed using a light microscope (Nikon Corporation).

Polyinosinic:polycytidylic acid [poly(I:C)] treatment of mUC-MSCs cell cultures. The TLR3-specific ligand, poly(I:C) was purchased from Sigma-Aldrich; Merck Millipore (cat no. P1530) and prepared according to the manufacturer's protocol. The cells were seeded at a density of $1.2 \times 10^{5}$ cells or $1.0 \times 10^{5}$ cells/well, respectively, in 6-well plates. The following day, medium was added either with or without poly(I:C). The treatment was continued for $24 \mathrm{~h}\left(1.2 \times 10^{5}\right.$ cells $)$ or $48 \mathrm{~h}$ $\left(1.0 \times 10^{5}\right.$ cells). The concentrations of poly(I:C) used were 0 , 10,25 and $50 \mu \mathrm{g} / \mathrm{ml}$.

RNA extraction, reverse transcription-polymerase chain reaction (RT-PCR) and RT-quantitative PCR (qPCR). Total RNA was extracted from the cells using TRIzol reagent (Invitrogen; Thermo Fisher Scientific, Inc.) and reverse transcribed into cDNA using the HiScript First-Strand cDNA Synthesis kit (cat no. R122-01; HiScript ${ }^{\circledR}$ Q RT SuperMix; Vazyme, Piscataway, NJ, USA) according to the manufacturer's protocol. Total RNA $(0.5 \mu \mathrm{g})$ was mixed with $2 \mu \mathrm{l}$ of $5 \mathrm{X}$ qRT SuperMix, and the thermocycling conditions were as follows: $25^{\circ} \mathrm{C}$ for $10 \mathrm{~min}$, $50^{\circ} \mathrm{C}$ for $30 \mathrm{~min}$ and $85^{\circ} \mathrm{C}$ for $5 \mathrm{~min}$. The RT-qPCR mixture contained $10 \mu \mathrm{l}$ of the SYBR-Green ${ }^{\circledR}$ Master Mix (2X; Bio-Rad Laboratories, Inc., Hercules, CA, USA) and $1 \mu \mathrm{l}$ of cDNA; the total volume was increased to $20 \mu \mathrm{l}$ with $\mathrm{ddH}_{2} \mathrm{O}$. The primer powder was fixed to the bottom of a 96-well plate and $20 \mu \mathrm{l}$ of the PCR mixture was added to each well. RT-qPCR was performed using the CFX96 real-time instrument (Bio-Rad Laboratories, Inc.) and the thermocycling conditions were as follows: $95^{\circ} \mathrm{C}$ for $5 \mathrm{~min}$, followed by 40 cycles of $95^{\circ} \mathrm{C}$ for $15 \mathrm{sec}$, annealing for $15 \mathrm{sec}$ and $72^{\circ} \mathrm{C}$ for $20 \mathrm{sec}$, and then a $65-95^{\circ} \mathrm{C}$ drawing dissociation curve. The sequences of the specific primers and the annealing temperature used for each are listed in Table I. The expression of each gene was defined from the threshold cycle and the melting temperatures were recorded. The relative changes in mRNA expression were analyzed using the $2^{-\Delta \Delta \mathrm{Cq}}$ method (25). $\beta$-actin was used as an internal control.

Western blot analysis. The cells were lysed and homogenized in RIPA buffer supplemented with protease inhibitors. Protein concentration was determined using a Bicinchoninic Acid Protein Quantification kit (CWBIO, Beijing, China), according to the manufacturer's instructions. Total proteins $(50 \mu \mathrm{g})$ were loaded on a $12 \%$ SDS-PAGE gel, followed by electrophoresis and transfer onto a PVDF membrane(EMD Millipore,Billerica, MA, USA). The sources and dilution ratios of primary antibodies were as follows: GAPDH (1:2,000; cat no. CW0100A; CWBIO), sex determining region Y-box 2 (Sox 2; 1:500; EMD Millipore), octamer-binding transcription factor 4 (Oct4; 1:200; cat no. sc-101534), Nanog (1:500; cat no. sc-293121) (both from Santa Cruz Biotechnology, Inc., Santa Cruz, CA, USA) and Spalt-like transcription factor 4 (Sall4; 1:500; cat no. ab31968; Abcam, Cambridge, UK). All primary antibodies were incubated overnight at $4^{\circ} \mathrm{C}$. The goat-anti-rabbit $\operatorname{IgG}$ (for Sox 2) and goat-anti-mouse IgG (for GAPDH, Oct4, Nanog and Sall4) horseradish peroxidase-conjugated secondary antibodies were purchased from CWBIO (dilution 1:2,000; cat nos. CW0103 and CW0102, respectively). All secondary antibodies were incubated for $1 \mathrm{~h}$ at $37^{\circ} \mathrm{C}$. The antigen-antibody complex was visualized using Immobilon Western Chemiluminescent HRP substrate (EMD Millipore).

Luminex assay. Following pre-treatment with poly(I:C) for 24 or $48 \mathrm{~h}$, the mUC-MSCs were washed and sequentially cultured in complete medium for another $48 \mathrm{~h}$. The supernatants were collected and centrifuged at $500 \mathrm{x} \mathrm{g}$ and $4{ }^{\circ} \mathrm{C}$ for $10 \mathrm{~min}$ to remove cell debris. A mouse cytokine/chemokine magnetic bead panel kit (cat no. MCYTOMAG-70K-12; EMD Millipore) was used to detect granulocyte colony stimulating factor (G-CSF), interferon- $\gamma($ IFN- $\gamma)$, interleukin (IL)-1 $\beta$, IL-6, IL-10, IL-15, IL-17, chemokine (C-X-C motif) ligand 10 (CXCL10), vascular endothelial growth factor (VEGF), and tumor necrosis factor- $\alpha$ (TNF- $\alpha$ ) in the prepared supernatant. All procedures were performed according to the manufacturer's protocol. The final detection and analysis were performed using the Luminex 200 system (EMD Millipore).

Statistical analysis. All data are presented as the mean \pm standard deviation. The statistical significances between two groups were analyzed using Student's t-test, whereas the differences among multiple groups were determined using one-way analysis of variance with GraphPad Prism 5 (GraphPad Software, Inc., La Jolla, CA, USA). A Tukey's post hoc test was used for multiple comparisons using SPSS version 20 (IBM Corp., Armonk, NY, USA). $\mathrm{P}<0.05$ was considered to indicate a statistically significant difference.

\section{Results}

Isolation of $m U C-M S C$ s from mouse UC. During the initial 24-48 h, the primary cells migrated out of the explants and adhered to the plastic surface, exhibiting heterogeneous morphology. At 3-5 days post-seeding, the cells began to form colonies (Fig. 1Aa). The well-developed colonies were trypsinized and passaged at a high density into a new cell culture dish. Following serial subculturing, a relatively homogeneous population of mUC-MSCs was observed at passages 5-7 when the purified cells exhibited spindle- or polygonal-shaped appearance (Fig. 1Ab), which was similar 
Table I. Primer sequences of target genes.

\begin{tabular}{|c|c|c|c|c|}
\hline Gene & $\begin{array}{c}\text { Primer } \\
\text { sequence }\left(5^{\prime}-3^{\prime}\right)\end{array}$ & $\begin{array}{l}\text { Fragment } \\
\text { size (bp) }\end{array}$ & $\begin{array}{c}\text { Annealing } \\
\text { temperature }\left({ }^{\circ} \mathrm{C}\right)\end{array}$ & $\begin{array}{c}\text { mRNA } \\
\text { accession no. }\end{array}$ \\
\hline \multirow[t]{2}{*}{ TLR3 } & F: GAGGTTGACGCACCTGTTCT & \multirow[t]{2}{*}{281} & \multirow[t]{2}{*}{60} & \multirow[t]{2}{*}{ NM_126166 } \\
\hline & R: GCTGCAGTCAGCTACGTTGT & & & \\
\hline \multirow[t]{2}{*}{ Runx2 } & F: AGCCTCTTCAGCGCAGTGAC & \multirow[t]{2}{*}{183} & \multirow[t]{2}{*}{63} & \multirow[t]{2}{*}{ XM_006523541 } \\
\hline & R: TGTTGTTGCTGCTGCTGTTG & & & \\
\hline \multirow[t]{2}{*}{ Adiponectin } & F: AGGAGATGCAGGTCTTCTTG & \multirow[t]{2}{*}{146} & \multirow[t]{2}{*}{58} & \multirow[t]{2}{*}{ NM_009605 } \\
\hline & R: CACTGAACGCTGAGCGATAC & & & \\
\hline \multirow[t]{2}{*}{ IL-6 } & F: AAGTCCGGAGAGGAGACTTC & \multirow[t]{2}{*}{487} & \multirow[t]{2}{*}{58} & \multirow[t]{2}{*}{ NM_031168 } \\
\hline & R: TGGATGGTCTTGGTCCTTAG & & & \\
\hline \multirow[t]{2}{*}{ IL-8 } & F: GGCTGTCCTTAACCTAGGCATCT & \multirow[t]{2}{*}{257} & \multirow[t]{2}{*}{63} & \multirow[t]{2}{*}{ NM_011339 } \\
\hline & R: GGTCCTCAGGTAGGAACCTGTTAGT & & & \\
\hline \multirow[t]{2}{*}{ CCL5 } & F: CATATGGCTCGGACACCAC & \multirow[t]{2}{*}{145} & \multirow[t]{2}{*}{62} & \multirow[t]{2}{*}{ NM_013653 } \\
\hline & R: CACACTTGGCGGTTCCTTC & & & \\
\hline \multirow[t]{2}{*}{ CXCL10 } & F: GCTGCAACTGCATCCATA & \multirow[t]{2}{*}{113} & \multirow[t]{2}{*}{57} & \multirow[t]{2}{*}{ NM_021274 } \\
\hline & Rev: CATCGTGGCAATGATCTC & & & \\
\hline \multirow[t]{2}{*}{$\beta$-actin } & F: CACGAAACTACCTTCAACTCC & \multirow[t]{2}{*}{265} & \multirow[t]{2}{*}{56} & \multirow[t]{2}{*}{ NM_001017992 } \\
\hline & R: CATACTCCTGCTTGCTGATC & & & \\
\hline
\end{tabular}

F, forward primer; R, reverse primer; TLR3, Toll-like receptor 3; Runx2, runt-related transcription factor 2; IL, interleukin; CCL5, chemokine (C-C motif) ligand 5; CXCL10, chemokine (C-X-C motif) ligand 10.

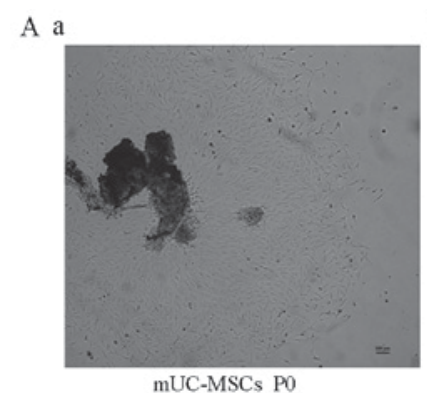

B

C

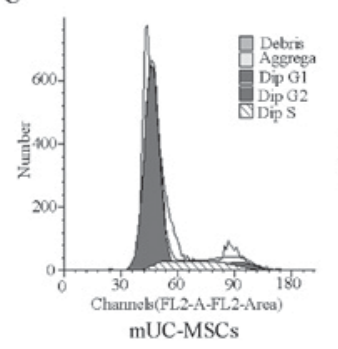

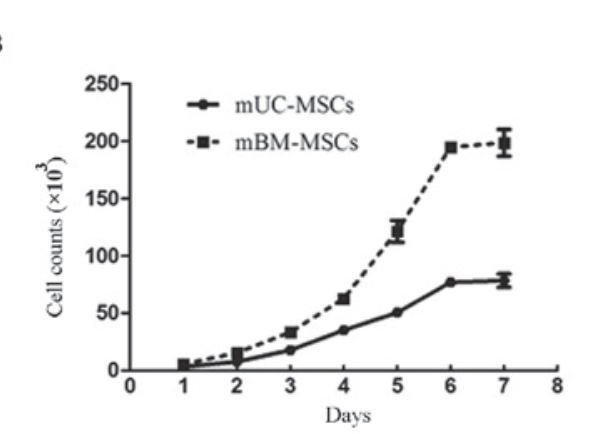

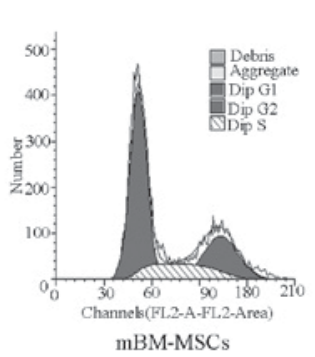

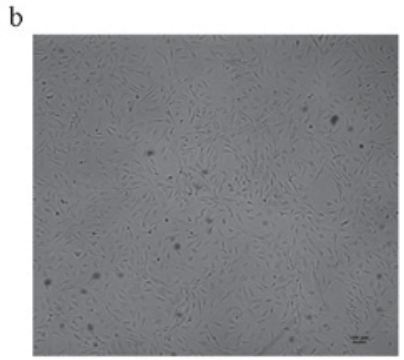

mUC-MSCs

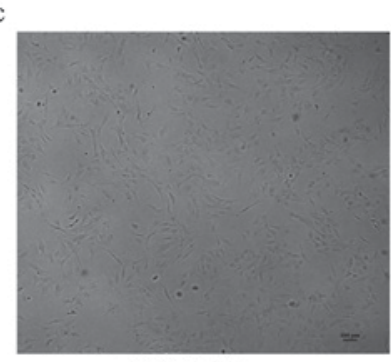

mBM-MSCs
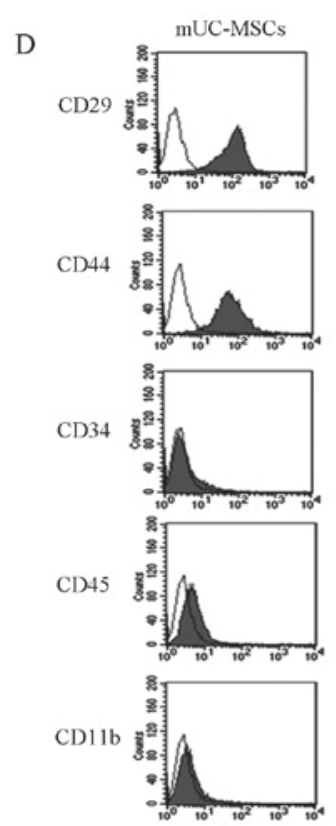
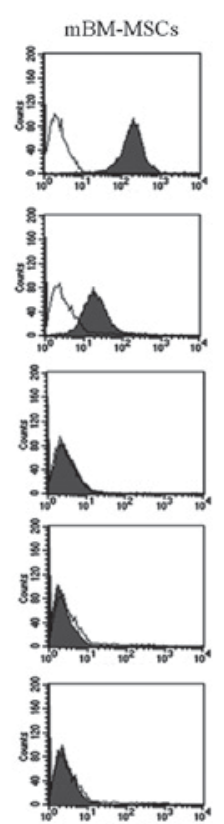

Figure 1. Morphology, growth, surface antigens and cell cycle of MSCs derived from mUC-MSCs and mBM-MSCs. (A) Appearance of mUC-MSCs and mBM-MSCs at passages (a) 0, (b) 10 and (c) 17 (magnification, x40; scale bar, $100 \mu \mathrm{m}$. (B) Growth curves of mUC-MSCs and mBM-MSCs. (C) Flow cytometric analysis of surface markers CD29, CD44, CD34, CD45 and CD11b in mUC-MSCs and mBM-MSCs. (D) DNA contents of mUC-MSCs and mBM-MSCs. mUC, mouse umbilical cord; mBM, mouse bone marrow; MSCs, mesenchymal stem cells; CD, cluster of differentiation. 
A

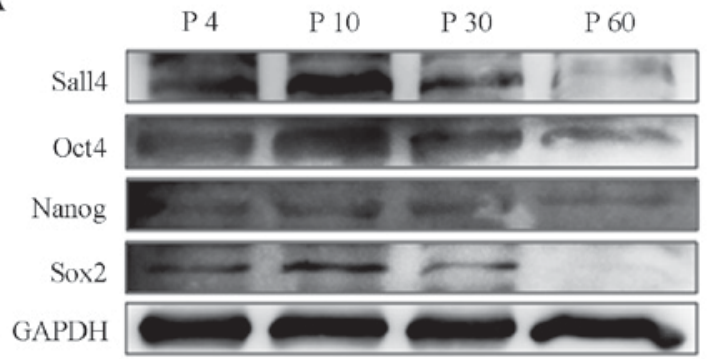

B

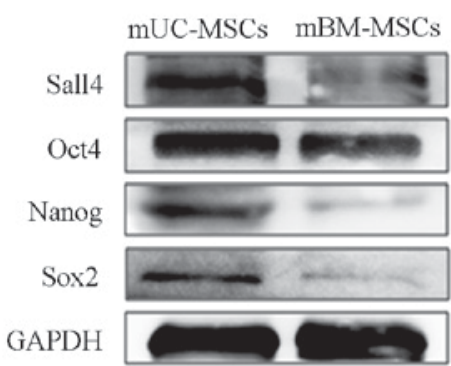

Figure 2. Stemness-related proteins in mUC-MSCs and mBM-MSCs. Western blot assay for the expression of Sox2, Nanog, Oct4 and Sall4 in (A) mUC-MSCs during long-term culture and (B) mBM-MSCs. mUC, mouse umbilical cord; mBM, mouse bone marrow; MSCs, mesenchymal stem cells; Sox2, Sex determining region Y-box 2; Oct4, octamer-binding transcription factor 4; Sall4, Spalt-like transcription factor 4.

to that of mBM-MSCs (Fig. 1Ac). No morphological changes were observed within 20 passages.

Comparison of growth characteristics between mUC-MSCs and $\mathrm{mBM}-\mathrm{MSCs}$. The mUC-MSCs and mBM-MSCs showed S-shaped growth curves (Fig. 1B). During the successive 7 days, the two types of cell exhibited a lag phase for 1 day and then moved into the logarithmic phase, when cells expanded rapidly. On day 6 , the cell counts of the two cell types reached their peak, followed by a plateau phase. The number of mUC-MSCs increased at a slower rate, compared with that of the mBM-MSCs. Similar results was observed in the cell cycle analysis. The DNA contents showed that the population of proliferating cells in the mUC-MSCs $(\mathrm{S}+\mathrm{G} 2 / \mathrm{M}$; $21.38 \%$ ) was smaller compared with that in the mBM-MSCs $(\mathrm{S}+\mathrm{G} 2 / \mathrm{M} ; 43.56 \%)$, although the subset of quiescent cells in the mUC-MSCs and mBM-MSCs (G0/G1; 78.62 and $56.44 \%$, respectively) accounted for the predominant population (Fig. 1C). Taken together, the mUC-MSCs possessed lower proliferative potential, compared with the mBM-MSCs.

Expression of surface antigens and stemness-related proteins in $m U C$-MSCs and $m B M-M S C s$. The results of the flow cytometric analysis revealed that the mUC-MSCs were positive for CD29 and CD44, but negative for CD34, CD45 and CD11b, similar to mBM-MSCs (Fig. 1D). To evaluate the potential of mUC-MSCs for long-term culture, the cells were subjected to continuous subculture 60 times. The cells of passages 4,10 , 30 and 60 were selected for evaluating the changes in protein expression levels of Sox 2, Nanog, Oct 4 and Sall4. The western blot analysis showed that the expression of these proteins markedly increased as the cells were purified (P10) and then decreased progressively with passages, indicating that purified mUC-MSCs of $<30$ passages were more suitable for further investigations (Fig. 2A). The mUC-MSCs exhibited higher expression levels of stemness-related proteins (Sox2, Nanog, Oct4 and Sall4), compared with the mBM-MSCs (Fig. 2B).

Differentiation potential of $m U C-M S C s$ and $m B M-M S C s$. As the mUC-MSCs exhibited significant differences in stemness-related proteins, compared with mBM-MSCs, the present study hypothesized that the two cell lines may have different differentiation potentials, particularly in adipogenic and osteogenic differentiation. Notably, the induction periods were shortened due to the higher efficiency of mBM-MSCs.
Although both the mUC-MSCs and mBM-MSCs were capable of differentiation into adipocytes or osteocytes, the former exhibited markedly lower potential for adipogenesis, as shown by positive staining of Oil red O (Fig. 3Aa) and the RT-qPCR analysis showing significantly lower mRNA levels of adiponectin in the differentiated mUC-MSCs, compared with those in the differentiated mBM-MSC (Fig. 3Ab). The same was true for osteogenesis, as shown by Oil red $\mathrm{O}$ (Fig. 3Ba) and the mRNA expression of runt-related transcription factor 2 (Runx2; Fig. 3Bb) being significantly lower in the differentiated mUC-MSCs, compared with those in the differentiated mBM-MSCs. These data indicated that mUC-MSCs possessed lower differentiation potential, compared with the mBM-MSCs.

Poly(I:C) enhances the expression of Sox2, Oct4 and Nanog in $m U C-M S C s$. It has been reported that poly(I:C) treatment can affect the migration, cytokine induction and immunosuppressive function of human MSCs $(26,27)$. However, the effect of poly(I:C) on mUC-MSCs remains to be elucidated. In the present study, the results of the RT-PCR analysis confirmed the expression of TLR3 in mUC-MSCs (Fig. 4A). To examine the effect of poly(I:C) on the levels of stemness-related proteins of mUC-MSCs, the cells were cultured with different doses of poly(I:C) for 24 or $48 \mathrm{~h}$. As shown in Fig. 4B, the mUC-MSCs in groups containing 10 or $25 \mu \mathrm{g} / \mathrm{ml}$ poly(I:C) exhibited higher levels of Sox 2, Oct4 and Nanog, compared with those in the control, whereas poly(I:C) at $50 \mu \mathrm{g} / \mathrm{ml}$ either failed to enhance the expression of these proteins $(24 \mathrm{~h})$ or increased their expression to a lesser extent $(48 \mathrm{~h})$.

Poly(I:C) promotes the expression and secretion of inflammatory cytokines in mUC-MSCs. Previous studies have indicated that TLR activation in MSCs can trigger the production of downstream cytokines. Therefore, a luminex assay was performed in the present study to determine the content of several inflammatory cytokines in the supernatants from mUC-MSCs pre-treated with poly(I:C), termed poly(I:C)-mUC-MSCs, including G-CSF, IFN- $\gamma$, IL-1 $\beta$, IL-6, IL-10, IL-15, IL-17, CXCL10, VEGF and TNF- $\alpha$. As shown in Fig. 5A (24 h) and Fig. 5B (48 h), IL-6 and CXCL10 were secreted at high levels and upregulated in the supernatants from the poly(I:C)-mUC-MSCs. In terms of concentration, $50 \mu \mathrm{g} / \mathrm{ml}$ poly(I:C) pre-treatment markedly increased the secretion of CXCL10, compared with that in the control, whereas IL-6 
A

a

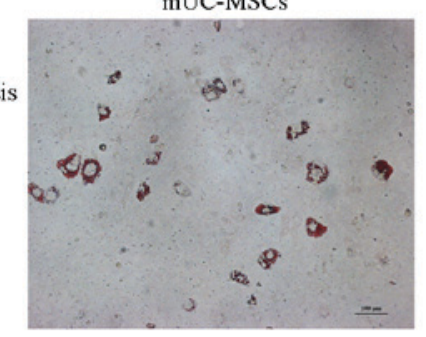

B

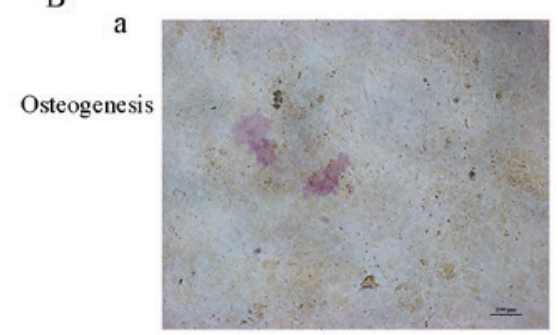

mBM-MSCs
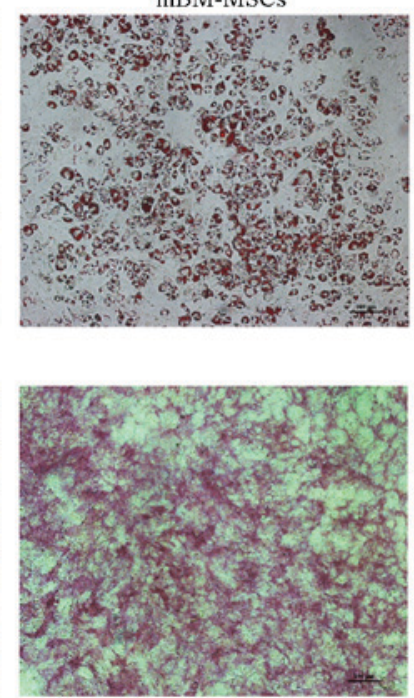

b

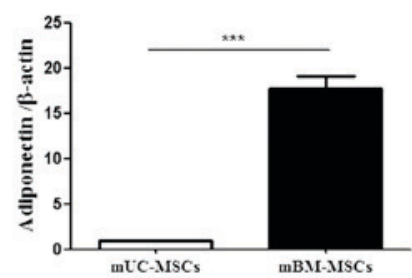

b

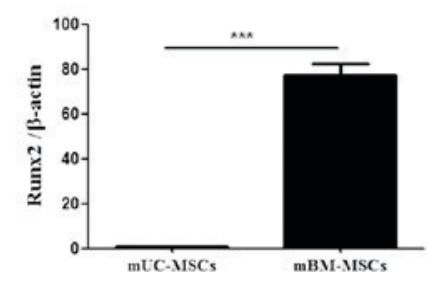

Figure 3. Adipogenic and osteogenic differentiation of mUC-MSCs and mBM-MSCs. (A) mUC-MSCs and mBM-MSCs were cultured in adipogenic induction medium for two cycles, followed by (a) Oil red O staining and (b) RT-qPCR analysis of mRNA levels of adiponectin. (B) mUC-MSCs and mBM-MSCs were cultured in osteogenic induction medium for four cycles, followed by (a) alkaline phosphatase staining (magnification, $\mathrm{x} 100$; scale bar, $100 \mu \mathrm{m})$ and (b) RT-qPCR analysis of mRNA levels of Runx2 ( $\left.{ }^{* * * *} \mathrm{P}<0.001\right)$. mUC, mouse umbilical cord; mBM, mouse bone marrow; MSCs, mesenchymal stem cells; RT-qPCR, reverse transcription-quantitative polymerase chain reaction; Runx2, runt-related transcription factor 2.
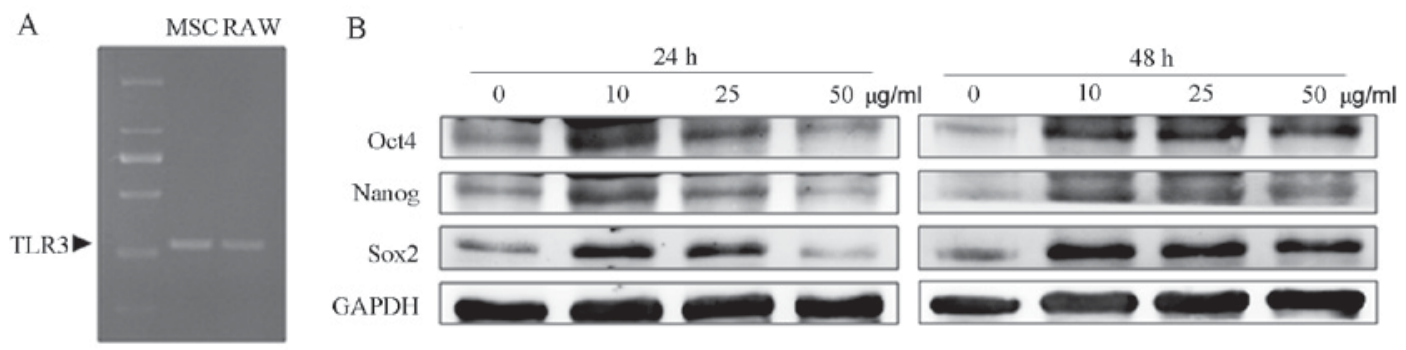

Figure 4. TLR3-specific ligand poly(I:C) increased the expression of stemness-related proteins in mUC-MSCs. (A) Reverse transcription-polymerase chain reaction analysis of the expression of TLR3 in mUC-MSCs. (B) Western blot analysis for the expression levels of Sox2, Nanog and Oct4 in mUC-MSCs pre-treated with $0,10,25$ and $50 \mu \mathrm{g} / \mathrm{ml}$ poly(I:C) for 24 or $48 \mathrm{~h}$. mUC, mouse umbilical cord; mBM, mouse bone marrow; MSCs, mesenchymal stem cells; poly(I:C), polyinosinic:polycytidylic acid; TLR3, Toll-like receptor 3; Sox2, Sex determining region Y-box 2; Oct4, octamer-binding transcription factor 4.

was altered to a lesser extent. To further confirm the enhanced production of IL-6 and CXCL10, RT-qPCR analysis was used to determine the mRNA levels. It was found that the upregulation of IL- 6 and CXCL10 were in accordance with the luminex assay data. CCL5 showed the most marked upregulation in mUC-MSCs pre-treated with $50 \mu \mathrm{g} / \mathrm{ml}$ poly(I:C); IL-8 was increased only in the mUC-MSCs pre-treated for 48 h (Fig. 5C).

\section{Discussion}

MSCs are considered the primary cell source for stem cell-based therapeutics. The use of UC-derived MSCs are increasing in popularity due to their unique properties. In preclinical studies, mMSCs have been most commonly used to establish experimental models, and transplantation of cross-species MSCs (i.e. human MSCs) remains the primary therapeutic method. However, xenogeneic immune responses may complicate the host cell responses (9) and further challenge the ability of MSCs to function across species barriers.
In consideration of allogeneic transplantation mimicry and mMSC deficiency, the present study successfully isolated MSCs from mUC using a novel method.

There are two primary methods to harvest MSCs, either from the whole UC or their compartments, including Wharton's jelly, cord lining, umbilical blood vessels and the perivascular region. These are the classic explant method and the enzymatic digestion method (7). Although the comparison between these two methods remains controversial, the former has been shown to be superior, with which plastic-adherent MSC-like cells were isolated from human gastric cancer tissues and adjacent non-cancerous tissues, and from hUC-MSCs $(28,29)$. In the present study, an MSC strain was obtained from the whole UC using an modified explant procedure and F12-containing media, which were referred to as mUC-MSCs according to previous reports $(7,10)$. The purified cells, which met the criteria of the International Society for Cellular Therapy (30), exhibited a fibroblast-like appearance, expressed typical surface antigens, differentiated into osteoblasts or adipocytes, and were maintained in a quiescent state, as indicated by 
A

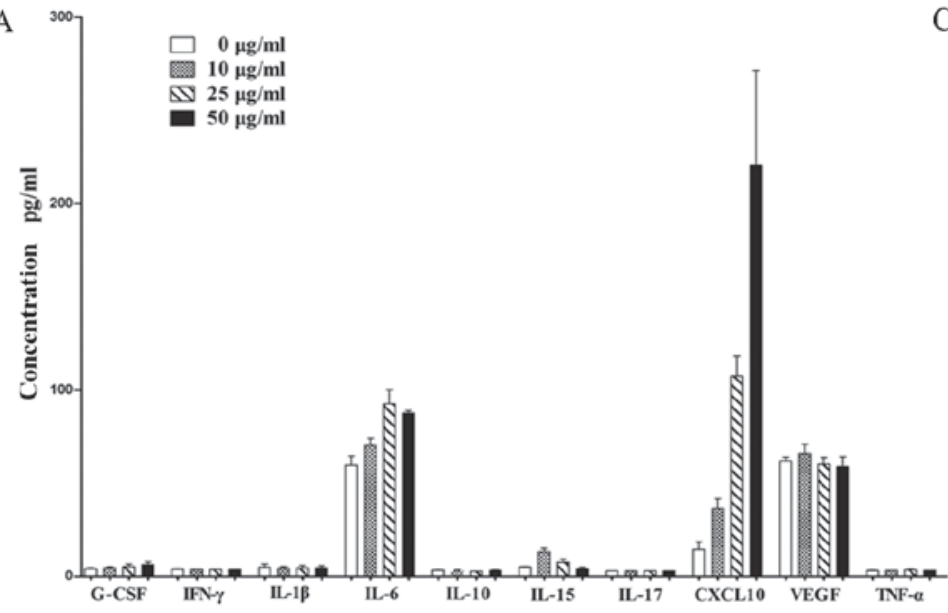

B

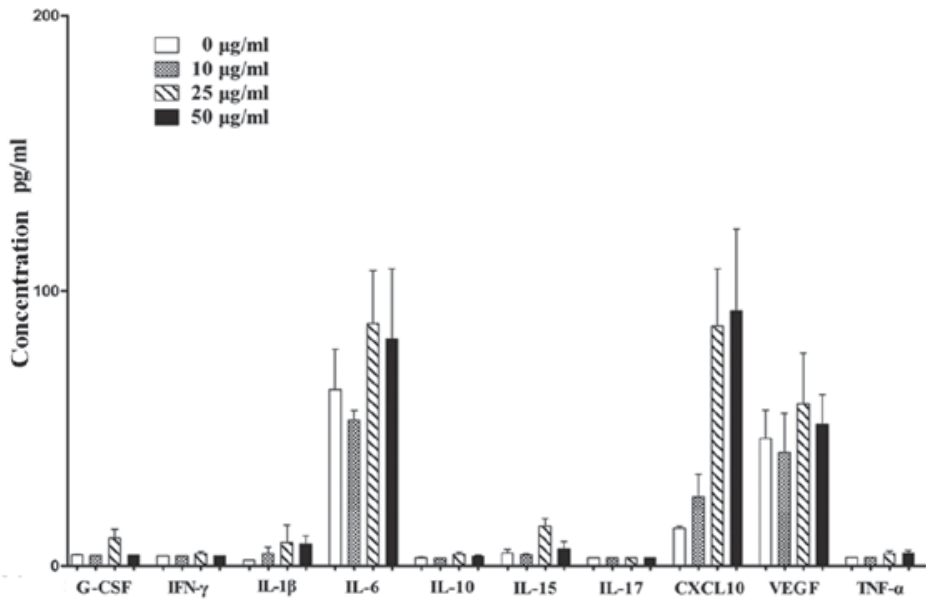

$\mathrm{C}$
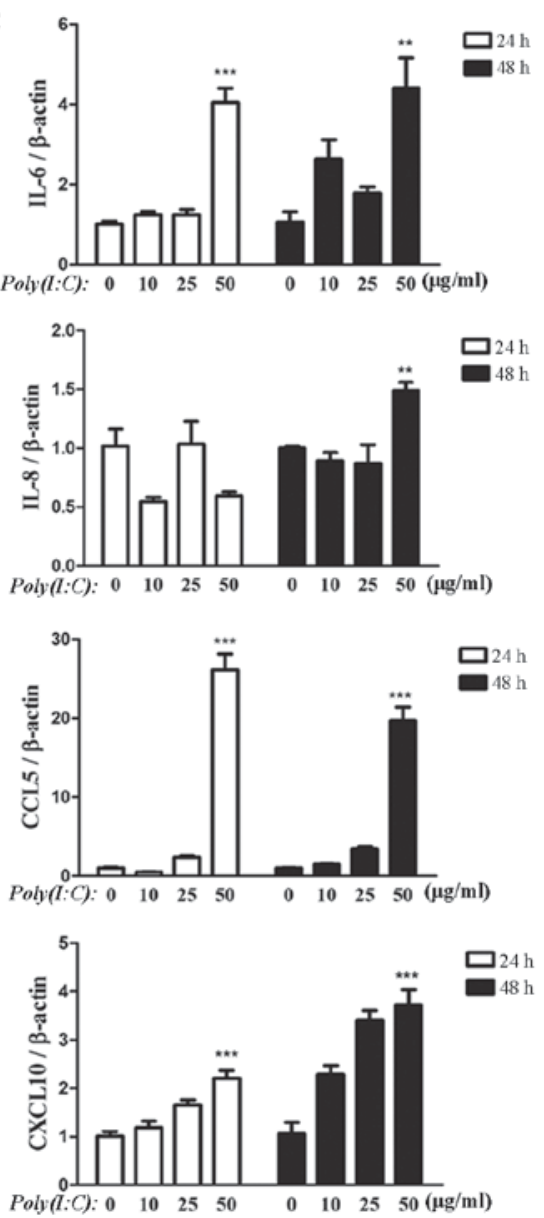

Figure 5. Poly(I:C) induces cytokine production in mUC-MSCs. A luminex assay of the levels of 10 cytokines in the supernatants from mUC-MSCs pre-treated with poly(I:C) for (A) $24 \mathrm{~h}$ or (B) $48 \mathrm{~h}$, including G-CSF, IFN- $\gamma$, IL-1 $\beta$, IL-6, IL-10, IL-15, IL-17, CXCL10, VEGF and TNF- $\alpha$. (C) Reverse transcription-quantitative polymerase chain reaction analysis of mRNA levels of IL-6, IL-8, CCL5 and CXCL10 in mUC-MSCs treated with poly(I:C) for 24 or $48 \mathrm{~h}$. ${ }^{* * * *} \mathrm{P}<0.001$ and ${ }^{* *} \mathrm{P}<0.01$, vs. control group. mUC-MSCs, mouse umbilical cord mesenchymal stem cells; G-CSF, granulocyte colony stimulating factor; IFN- $\gamma$, interferon- $\gamma$; IL, interleukin; CXCL10, chemokine (C-X-C motif) ligand 10; VEGF, vascular endothelial growth factor; TNF- $\alpha$, tumor necrosis factor- $\alpha$; CCL5, chemokine (C-C motif) ligand 5; poly(I:C), polyinosinic:polycytidylic acid.

the high proportion of cells in the G0/G1 phase. In addition, the expression of stemness-related proteins, including Sox2, Nanog, Oct4 and Sall4, were enhanced in purified mUC-MSCs and gradually decreased with passages, indicating their stemness maintenance ability during long-term culture. The abovementioned data suggested that the modified method used in the present study to isolate and culture mUC-MSCs, free from chemical injury and with the addition of nutritional factors, is efficient, reliable and cost-saving.

Although mMSCs can be derived from various tissues (4), $\mathrm{BM}$ remains the most commonly used tissue source. MSCs from BM are limited in terms of cell number, whereas MSCs account for the predominant stem cell population in the UC (10). Therefore, investigation of the characteristics of mUC-MSCs relative to the 'gold standard' cell source of mBM-MSCs was required. The present study found that $\mathrm{mBM}$-MSCs under the adherent method required a longer duration for purification, of at least 13 passages. The two cell populations presented with similar morphology and, to a certain extent, surface antigen profile. With the exception of the five essential markers, it has been reported that the differential expression of SSEA-4, LNGFR, CD56 and CD146 can distinguish hUC-MSCs from
hBM-MSCs (31). The growth curve assay and DNA content analysis in the present study indicated that mUC-MSCs possessed a lower proliferative potential, compared with the mBM-MSCs. However, similar comparison within hMSCs showed the opposite result, namely that hUC-MSC showed optimal proliferation, which may due to certain abundant genes involved in cell proliferation $(7,32,33)$. To compare the protein expression levels between mUC-MSCs and mBM-MSCs, the present study focused on stemness-related proteins, including Sox 2, Nanog, Oct 4 and Sall4. The results revealed that the levels of these proteins were higher in the mUC-MSCs, suggesting that the cells were primitive stem cells $(10,33)$. The present study found that mUC-MSCs presented with markedly lower adipogenic and osteogenic differentiation efficiencies, which was in accordance with previous reports involving human and enquine MSCs (11,32-34). The present study is the first, to the best of our knowledge, to report on the similarities and differences between mUC-MSCs and mBM-MSCs, and indicated that the reasonable selection of mMSCs be made based on their own advantages and the relevant models. Considering the differences, further investigations are required to clarify the respective mechanisms. 
Previous studies have confirmed that MSCs express functional TLR3 and that its activation regulates MSC functions (22-26). The present study confirmed the presence of TLR3 in mUC-MSCs, in accordance with mBM-MSCs (22). Subsequently, a preliminary experiment was performed to determine the effect of poly(I:C), the TLR3 agonist, on mUC-MSCs. The enhanced expression of stemness-related proteins (Sox2, Oct4 and Nanog) was observed in the poly(I:C)-treated mUC-MSCs, which was similar to the data from a previous study suggesting the potential involvement of TLR3 in stemness maintenance of MSCs (35). As TLR signaling is closely associated with inflammatory cytokines, the secretion and mRNA levels of several cytokines were detected in the mUC-MSCs treated with poly(I:C). The results showed that the levels of IL-6, CXCL10, CCL5 and IL-8 were markedly upregulated. According to previous repots, these cytokines may be involved in immune modulation and cancer progression $(23,24)$.

In conclusion, the present study focused on a novel cell line of MSCs from mouse UC using a novel method. This, to the best of our knowledge, is the first demonstration of comparative analysis of mUC-MSCs and their responses to TLR3 activation. It was found that the mUC-MSCs exhibited MSC-like characteristics and shared similarities with mBM-MS, however, they exhibited differences in purification, proliferation, stem cell markers and differentiation. Poly(I:C) increased the expression of stemness-related proteins and inflammatory cytokines. Further investigations are required to confirm the manifestation and investigate the underlying mechanisms. The results of the present study provide novel evidence for the selection of mMSCs and offer further insight into the role of TLR3 in the regulation of mMSCs.

\section{Acknowledgements}

This study was supported by the National Natural Science Foundation of China (grant nos. 31340040, 81272481, 81270214 and 81572075$)$, the Jiangsu Province for Outstanding Sci-tech Innovation Team in Colleges and Universities (grant no. SJK2013-10), the Jiangsu Province's Outstanding Medical Academic Leader and Sci-tech Innovation Team Program (grant no. LJ201117) and the Special Funded Projects of National Postdoctoral Fund (grant no. 2017T100337).

\section{References}

1. Friedenstein AJ, Chailakhjan RK and Lalykina KS: The development of fibroblast colonies in monolayer cultures of guinea-pig bone marrow and spleen cells. Cell Tissue Kinet 3: 393-403, 1970.

2. Caplan AI: Mesenchymal stem cells. J Orthop Res 9: 641-650, 1991.

3. Frausin S, Viventi S, Verga Falzacappa L, Quattromani MJ, Leanza G, Tommasini A and Valencic E: Wharton's jelly derived mesenchymal stromal cells: Biological properties, induction of neuronal phenotype and current applications in neurodegeneration research. Acta Histochem 117: 329-338, 2015.

4. Iser IC, Bracco PA, Goncalves CE, Zanin RF, Nardi NB, Lenz G, Battastini AM and Wink MR: Mesenchymal stem cells from different murine tissues have differential capacity to metabolize extracellular nucleotides. J Cell Biochem 115: 1673-1682, 2014.

5. Jung J, Choi JH, Lee Y, Park JW, Oh IH, Hwang SG, Kim KS and Kim GJ: Human placenta-derived mesenchymal stem cells promote hepatic regeneration in $\mathrm{CCl} 4$-injured rat liver model via increased autophagic mechanism. Stem Cells 31: 1584-1596, 2013.
6. Park M, Kim YH, Woo SY, Lee HJ, Yu Y, Kim HS, Park YS, Jo I, Park JW, Jung SC, et al: Tonsil-derived mesenchymal stem cells ameliorate CCl4-induced liver fibrosis in mice via autophagy activation. Sci Rep 5: 8616, 2015.

7. Nagamura-Inoue $\mathrm{T}$ and $\mathrm{He} \mathrm{H}$ : Umbilical cord-derived mesenchymal stem cells: Their advantages and potential clinical utility. World J Stem Cells 6: 195-202, 2014.

8. Harris DT: Umbilical cord tissue mesenchymal stem cells: Characterization and clinical applications. Curr Stem Cell Res Ther 8: 394-399, 2013.

9. Li WW, Wei YH, Li H, Lai DM and Lin TN: Isolation and characterization of a novel strain of mesenchymal stem cells from mouse umbilical cord: Potential application in cell-based therapy. PLoS One 8: e74478, 2013.

10. Bongso A and Fong CY: The therapeutic potential, challenges and future clinical directions of stem cells from the wharton's jelly of the human umbilical cord. Stem Cell Rev 9: 226-240, 2013.

11. Barberini DJ, Freitas NP, Magnoni MS, Maia L, Listoni AJ, Heckler MC, Sudano MJ, Golim MA, da Cruz Landim-Alvarenga $F$ and Amorim RM: Equine mesenchymal stem cells from bone marrow, adipose tissue and umbilical cord: Immunophenotypic characterization and differentiation potential. Stem Cell Res Ther 5: 25, 2014.

12. Fink KD, Rossignol J, Crane AT, Davis KK, Bombard MC, Bavar AM, Clerc S, Lowrance SA, Song C, Lescaudron L and Dunbar GL: Transplantation of umbilical cord-derived mesenchymal stem cells into the striata of R6/2 mice: Behavioral and neuropathological analysis. Stem Cell Res Ther 4: 130, 2013.

13. Mitchell KE, Weiss ML, Mitchell BM, Martin P, Davis D, Morales L, Helwig B, Beerenstrauch M, Abou-Easa K, Hildreth T, et al: Matrix cells from wharton's jelly form neurons and glia. Stem Cells 21: 50-60, 2003.

14. Ganta C, Chiyo D, Ayuzawa R, Rachakatla R, Pyle M, Andrews G, Weiss M, Tamura M and Troyer D: Rat umbilical cord stem cells completely abolish rat mammary carcinomas with no evidence of metastasis or recurrence 100 days post-tumor cell inoculation. Cancer Res 69: 1815-1820, 2009.

15. Liang J, Gu F, Wang H, Hua B, Hou Y, Shi S, Lu L and Sun L: Mesenchymal stem cell transplantation for diffuse alveolar hemorrhage in SLE. Nat Rev Rheumatol 6: 486-489, 2010.

16. Lindenmair A, Hatlapatka T, Kollwig G, Hennerbichler S, Gabriel C, Wolbank S, Redl H and Kasper C: Mesenchymal stem or stromal cells from amnion and umbilical cord tissue and their potential for clinical applications. Cells 1: 1061-1088, 2012.

17. Li W, Zhang Q, Wang M, Wu H, Mao F, Zhang B, Ji R, Gao S, Sun Z, Zhu W, et al: Macrophages are involved in the protective role of human umbilical cord-derived stromal cells in renal ischemia-reperfusion injury. Stem Cell Res 10: 405-416, 2013.

18. Wang H, Qiu X, Ni P, Qiu X, Lin X, Wu W, Xie L, Lin L, Min J, Lai X, et al: Immunological characteristics of human umbilical cord mesenchymal stem cells and the therapeutic effects of their transplantion on hyperglycemia in diabetic rats. Int $\mathrm{J}$ Mol Med 33: 263-270, 2014.

19. Li J, Ezzelarab MB and Cooper DK: Do mesenchymal stem cells function across species barriers? Relevance for xenotransplantation. Xenotransplantation 19: 273-285, 2012.

20. Bernardo ME and Fibbe WE: Mesenchymal stromal cells: Sensors and switchers of inflammation. Cell Stem Cell 13: 392-402, 2013.

21. DelaRosa O and Lombardo E: Modulation of adult mesenchymal stem cells activity by toll-like receptors: Implications on therapeutic potential. Mediators Inflamm 2010: 865601, 2010.

22. Pevsner-Fischer M, Morad V, Cohen-Sfady M, Rousso-Noori L, Zanin-Zhorov A, Cohen S, Cohen IR and Zipori D: Toll-like receptors and their ligands control mesenchymal stem cell functions. Blood 109: 1422-1432, 2007.

23. Waterman RS, Tomchuck SL, Henkle SL and Betancourt AM: A new mesenchymal stem cell (MSC) paradigm: Polarization into a pro-inflammatory MSC1 or an immunosuppressive MSC2 phenotype. PLoS One 5: e10088, 2010.

24. Waterman RS, Henkle SL and Betancourt AM: Mesenchymal stem cell 1 (MSC1)-based therapy attenuates tumor growth whereas MSC2-treatment promotes tumor growth and metastasis. PLoS One 7: e45590, 2012.

25. Livak KJ and Schmittgen TD: Analysis of relative gene expression data using real time quantitative PCR and the 2(Delta Delta $\mathrm{C}(\mathrm{T})$ ) method. Methods 25: 402-408, 2001. 
26. Tomchuck SL, Zwezdaryk KJ, Coffelt SB, Waterman RS, Danka ES and Scandurro AB: Toll-like receptors on human mesenchymal stem cells drive their migration and immunomodulating responses. Stem Cells 26: 99-107, 2008.

27. Zhao X, Liu D, Gong W, Zhao G, Liu L, Yang L and Hou Y: The toll-like receptor 3 ligand, poly(I:C), improves immunosuppressive function and therapeutic effect of mesenchymal stem cells on sepsis via inhibiting miR-143. Stem Cells 32: 521-533, 2014

28. Xu X, Zhang X, Wang S, Qian H, Zhu W, Cao H, Wang M, Chen Y and $\mathrm{Xu} \mathrm{W}$ : Isolation and comparison of mesenchymal stem-like cells from human gastric cancer and adjacent non-cancerous tissues. J Cancer Res Clin Oncol 137: 495-504, 2011.

29. Qiao C, Xu W, Zhu W, Hu J, Qian H, Yin Q, Jiang R, Yan Y, Mao F, Yang H, et al: Human mesenchymal stem cells isolated from the umbilical cord. Cell Biol Int 32: 8-15, 2008.

30. Dominici M, Le Blanc K, Mueller I, Slaper-Cortenbach I Marini F, Krause D, Deans R, Keating A, Prockop Dj and Horwitz E: Minimal criteria for defining multipotent mesenchymal stromal cells. The international society for cellular therapy position statement. Cytotherapy 8: 315-317, 2006.

31. Zeddou M, Relic B and Malaise MG: Umbilical cord fibroblasts: Could they be considered as mesenchymal stem cells. World J Stem Cells 6: 367-370, 2014.
32. Hsieh JY, Fu YS, Chang SJ, Tsuang YH and Wang HW: Functional module analysis reveals differential osteogenic and stemness potentials in human mesenchymal stem cells from bone marrow and Wharton's jelly of umbilical cord. Stem Cells Dev 19: 1895-1910, 2010.

33. Hua J, Gong J, Meng H, Xu B, Yao L, Qian M, He Z, Zou S, Zhou B and Song Z: Comparison of different methods for the isolation of mesenchymal stem cells from umbilical cord matrix: Proliferation and multilineage differentiation as compared to mesenchymal stem cells from umbilical cord blood and bone marrow. Cell Biol Int: Oct 7, 2013 (Epub ahead of print). doi: 10.1002/cbin.10188.

34. Ding DC, Chang YH, Shyu WC and Lin SZ: Human umbilical cord mesenchymal stem cells: A new era for stem cell therapy. Cell Transplant 24: 339-347, 2015.

35. Zhang L, Liu D, Pu D, Wang Y, Li L, He Y, Li Y, Li L, Qiu Z, Zhao $\mathrm{S}$ and $\mathrm{Li} \mathrm{W}$ : The role of toll-like receptor 3 and 4 in regulating the function of mesenchymal stem cells isolated from umbilical cord. Int J Mol Med 35: 1003-1010, 2015. International (CC BY-NC-ND 4.0) License. 